\title{
Echocardiographic Evaluation and Management of Right Ventricular Dysfunction during Cardiac Transplantation
}

\author{
${ }^{1}$ Alok Kumar, ${ }^{2}$ VK Arya, ${ }^{3}$ Ajay Kumar, ${ }^{4}$ Goverdhan Dutt Puri, ${ }^{5} \mathrm{KST}$ Shyam, ${ }^{6}$ Ajay Bahl
}

\section{ABSTRACT}

Cardiac allotransplantation has, over the years, become the established therapeutic modality for patients with end-stage heart failure. This case report focuses on the anesthetic challenges involved in the perioperative management of recipient with right ventricular dysfunction as well as echocardiographic assessment to assist in therapy.

Keywords: Cardiac transplant, Echocardiography, Right ventricle dysfunction.

How to cite this article: Kumar A, Arya VK, Kumar A, Puri GD, Shyam KST, Bahl A. Echocardiographic Evaluation and Management of Right Ventricular Dysfunction during Cardiac Transplantation. J Perioper Echocardiogr 2016;4(1):10-13.

\section{Source of support: Nil}

Conflict of interest: None

\section{INTRODUCTION}

Right ventricle (RV) dysfunction is a leading cause of mortality (19\%) and morbidity (50\%) after cardiac transplant. ${ }^{1}$ Improved techniques of preserving donor heart, significant advances in immunosuppressive therapy, inhaled nitric oxide, and enhanced role of echocardiography in perioperative period have dramatically improved the outcome of heart transplantation over the past four decades. Identification of such dysfunction early and their prompt management is the key to successful recovery.

\footnotetext{
${ }^{1,3}$ Senior Resident, ${ }^{2,4}$ Professor, ${ }^{5}$ Professor and Head ${ }^{6}$ Additional Professor

${ }^{1}$ Department of Cardiac Anaesthesia, Postgraduate Institute of Medical Education and Research, Chandigarh, India

${ }^{2-4}$ Department of Anaesthesia and Intensive Care, Postgraduate Institute of Medical Education and Research, Chandigarh India

${ }^{5}$ Department of Cardiovascular and Thoracic Surgery Postgraduate Institute of Medical Education and Research Chandigarh, India

${ }^{6}$ Department of Cardiology, Postgraduate Institute of Medical Education and Research, Chandigarh, India
}

Corresponding Author: Alok Kumar, Senior Resident Department of Cardiac Anaesthesia, Postgraduate Institute of Medical Education and Research, Chandigarh, India, Phone: +918146044104, e-mail: docsomi@yahoo.com

\section{CASE REPORT}

\section{Donor Details}

The donor was a 16-year-old boy admitted after road traffic accident. Predonation echocardiography of donor heart showed left ventricle ejection fraction (LVEF) of $65 \%$, mild RV dilatation, and mild tricuspid regurgitation (TR) with estimated pulmonary artery systolic pressure (PASP) of $30 \mathrm{~mm} \mathrm{Hg}$, tricuspid annular plane systolic excursion (TAPSE) of $15 \mathrm{~mm}$. Patient hemodynamics was stabilized with judicious fluid management and ionotrope (dobutamine). After evaluation patient was accepted as donor for heart transplant.

\section{Recipient Condition}

The recipient of the cardiac transplant was a 14-year-old male child diagnosed with end-stage cardiac disease secondary to idiopathic dilated cardiomyopathy (DCMP). The patient had New York Heart Association (NYHA) class 4 symptoms despite maximal medical management with preserved liver and renal functions. On investigation, chest X-ray showed cardiomegaly with cardiothoracic ratio of 0.8 , and electrocardiogram showed normal sinus rhythm. Two-dimensional transthoracic echocardiography (2D TTE) echocardiography confirmed DCMP with ejection fraction (EF) of 10 to $15 \%$, trivial mitral regurgitation (MR), moderate TR, and mild pulmonary arterial hypertension with pulmonary vascular resistance index (PVRI) of 352 dynes sec $/ \mathrm{cm}^{5} / \mathrm{m}^{2}$. Multigated acquisition scan showed right ventricle ejection fraction (RVEF) $32 \%$ and LVEF $12 \%$ with dilated LV. Cath angiogram revealed normal coronaries with right dominance and LV end diastolic pressure of $30 \mathrm{~mm} \mathrm{Hg}$.

We followed standard management of organ donation including invasive monitoring, antibiotics, and immunosuppression with intraoperative methylprednisolone. Similarly, standard surgical procedure of heart retrieval was followed. Organ preservation was achieved with standard custodiol solution in vivo and with warm blood after transplantation. Cold ischemia time was 20 minutes as organ was transferred within the same hospital. Although aortic cross clamp was only 63 minutes, patient had to be supported on cardiopulmonary bypass for 6 hours due to severe biventricular dysfunction and 
gradually separated. Patient hemodynamics was monitored using transesophageal echocardiography (TEE). After transplant, patient had biventricular severe dysfunction (LVEF 30-35\%) with moderate to severe TR and mild MR. Right ventricular systolic pressure (RVSP) was $41 \mathrm{~mm} \mathrm{Hg}$ and PVRI was 132.5 dynes sec $/ \mathrm{cm}^{5} / \mathrm{m}^{2}$ with interventricular septal flattening and moderate RV dilatation. Pulmonary artery pressures were $38 / 16 \mathrm{~mm} \mathrm{Hg}$. There was no anastomotic leak or narrowing of any anastomotic point observed after the surgery.

Postoperative course in the intensive care unit (ICU) was complicated with persistent RV dysfunction which was reflected on TEE by dilated and hypokinectic RV sharing the apex, RV TAPSE of $7 \mathrm{~mm}$, and severe TR with tricuspid annular dilatation (RVSP $=40 \mathrm{~mm} \mathrm{Hg}$ ). There was associated moderate LV dysfunction. This was managed with ionotropes and judicious fluid balance. Patient was received in ICU from the operating room with adrenaline $0.15 \mu \mathrm{g} / \mathrm{kg} / \mathrm{min}$, nor adrenaline $0.1 \mu \mathrm{g} / \mathrm{kg} / \mathrm{min}$, dobutamine $10 \mu \mathrm{g} / \mathrm{kg} / \mathrm{min}$, milrinone $0.5 \mu \mathrm{g} / \mathrm{kg} / \mathrm{min}$. Inhaled nitric oxide (NO) was started in ICU at $25 \mathrm{ppm}$. Diuretics (furosemide intravenous as and when required) were given to keep target central venous pressure $(\mathrm{CVP})<10 \mathrm{~cm} \mathrm{H}_{2} \mathrm{O}$ with mean arterial pressure (MAP) $>70 \mathrm{~mm} \mathrm{Hg}$. On TTE global longitudinal strain (GLS) and free wall RV strain of -8 and $-8.7 \%$ respectively.

On the 2nd day LV function improved; however, RV dysfunction persisted. Epinephrine was tapered and vasopressin was started. The patient was managed with nor epinephrine, milrinone, and dobutamine infusion. Inhaled NO was continued, with time RV volume and TR severity regressed (RVSP $=36 \mathrm{~mm} \mathrm{Hg}$ ). Pulmonary artery pressures were $30 / 16 \mathrm{~mm} \mathrm{Hg}$ at this stage. The patient was weaned and extubated as he gradually improved. The patient was continued on dubutamine and milrinone infusion on in view of RV dysfunction which were gradually tapered and discontinued by day 5 while oral sildenafil $25 \mathrm{mg}$ thrice a day was started on day 2. Oral torsemide $10 \mathrm{mg}$ and spirinolactone $12.5 \mathrm{mg}$ once a day were added at this point as CVP gradually fell from 16 to $<10$ while hemodynamics were maintained on spontaneous respiration.

Serial 2D TTE, color-flow imaging, and strain measurements were obtained in multiple views acquired on days $4,7,14,21,28$, and 35 postoperatively. With passage of time, RV free wall thickness gradually increased. Right ventricle also showed gradual improvement which correlated well with GLS and free wall RV strain measurements (Fig. 1). The patient had TR postoperatively which gradually decreased in severity. The LVEF remained consistently above $60 \%$ while GLS of LV showed gradual improvement with average of $10.25 \%$. Tricuspid annular

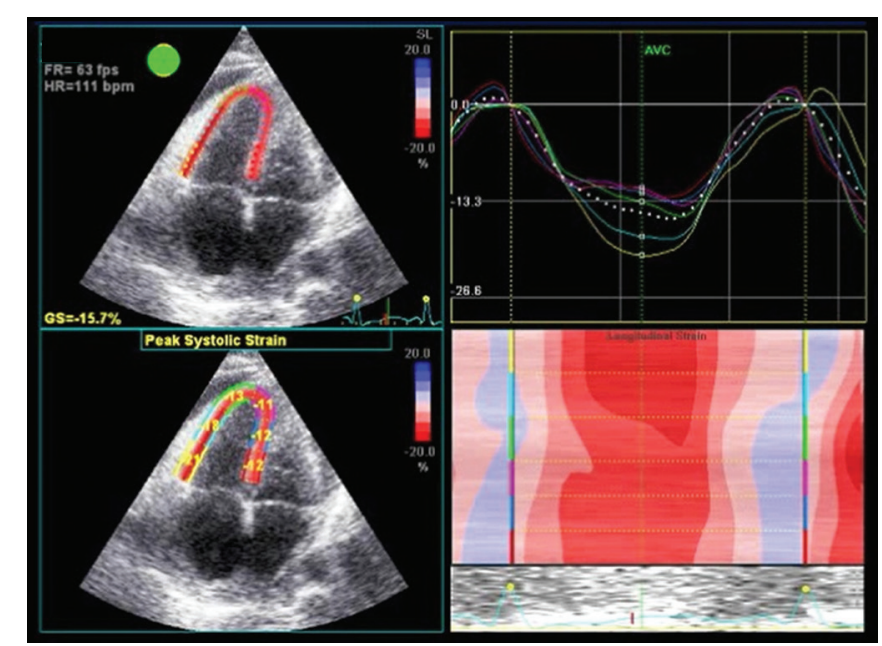

Fig. 1: Two-dimensional transthoracic speckle tracking right ventricle strain on day 28 posttransplant

plane systolic excursion increased from 7 to 9, lateral tricuspid annulus velocity $\left(\mathrm{s}^{\prime}\right)$ showed improvement from 6 to $11 \mathrm{~m} / \mathrm{s}$, while isovolumic acceleration (IVA) time remained between 1.8 and $3 \mathrm{~m} / \mathrm{s}^{2}$. Diastolic function was assessed for LV which also showed gradual improvement (Table 1). The patient received the standard tripleregimen immunosuppressive therapy postoperatively after transplantation.

\section{DISCUSSION}

Right ventricle dysfunction in the immediate postcardiac transplant period has been described, although LV systolic function remains normal in most patients, which unless aggressively treated may progress to overt RV failure. ${ }^{1-4}$ Various mechanisms for RV dysfunction may include donor heart dysfunction, prolonged ischemic time, preexisting pulmonary hypertension in the recipient, or immediate rejection of the transplanted heart.

The effect of brain death and subsequent hypothermic cardioplegic arrest and storage on subsequent posttransplant RV function has been examined. Brain death has a significant impact on cardiac function in the organ donor, and presumably influences subsequent ventricular performance after implantation in the recipient. After 6 hours of brain death, ventricular systolic function and contractility significantly decreases. This decrease is more for the RV than for the left ventricle (LV). The etiology and mechanism of the deterioration in RV (and LV) function associated with the onset of brain death appears to be related to the severe hyperdynamic and hypertensive state associated with the catecholamine release that accompanies acute increase in intracranial pressure. ${ }^{5}$ The donor heart in our case did show RV dysfunction which was managed with inotropes and fluids. The period of heart storage and transport is the second period that affects posttransplant function. Currently, most hearts are 
Table 1: Two-dimensional transthoracic echocardiographic parameters at days 4, 7, 14, 21, 28, and 35 posttransplant

\begin{tabular}{|c|c|c|c|c|c|c|}
\hline Posttransplant Day ... & 4 & 7 & 14 & 21 & 28 & 35 \\
\hline LVEF (\%) & 66 & 68 & 68 & 54 & 61 & 65 \\
\hline GLS-LV (\%) & 9.9 & 8.4 & 11.3 & 8.7 & 14.4 & 15 \\
\hline $\mathrm{E} / \mathrm{A}$ & 2.84 & 2.05 & 1.8 & 1.22 & 1.2 & 1.15 \\
\hline E/e' (septal) & 17 & 11 & 11 & 10.5 & 7.2 & 7 \\
\hline IVRT (ms) & 285 & 292 & 307 & 318 & 307 & 315 \\
\hline DT (ms) & 148 & 159 & 160 & 180 & 180 & 181 \\
\hline LV post wall thickness (mm) & 15 & 13 & 13 & 13 & 12 & 12 \\
\hline Septum thickness (mm) & 11 & 13 & 13 & 13 & 15 & 15 \\
\hline RV-S' (m/s) & 6 & 8 & 8 & 9 & 11 & 12 \\
\hline RV-IVA $\left(\mathrm{m} / \mathrm{s}^{2}\right)$ & 3 & 1.8 & 2.4 & 2.9 & 3.1 & 3.1 \\
\hline GLS RV (\%) & 8.8 & 12.3 & 12.8 & 14.8 & 15.7 & 17.4 \\
\hline Free wall RV (\%) & 9 & 11.3 & 12.3 & 14 & 17.3 & 18.3 \\
\hline $\mathrm{TR} \max (\mathrm{m} / \mathrm{s})$ & 2.8 & 2.64 & 2.49 & 2.5 & 2.5 & 2.4 \\
\hline TR PG (mm Hg) & 31.3 & 29 & 25 & 25 & 25 & 24 \\
\hline RV free wall thickness (mm) & 9.8 & 11.6 & 12.3 & 12.3 & 12.8 & 11 \\
\hline TAPSE $(\mathrm{mm})$ & 7 & 8 & 8 & 9 & 9 & 12 \\
\hline
\end{tabular}

LVEF: Left ventricle ejection fraction; GLS: Global longitudinal strain; IVRT: Isovolumic relaxation time; DT: Deceleration time; IVA: Isovolumic acceleration; TR: Tricuspid regurgitation; PG: Pressure gradient; RV: Right ventricle; TAPSE: Tricuspid annular plane systolic excursion

stored and transported in cold cardioplegic preservation solutions. ${ }^{6}$ The cold and warm ischemic time in our case was very short as the heart had to be transported in the same hospital.

Speckle-tracking has many advantages in comparison with tissue Doppler imaging (TDI), such as deformation analysis in 2D, tissue movement assessment independent of adjacent segments, angle independence, and better spatial resolution. The LV global longitudinal strain $(<15.5 \%)$ identifies patients at low risk for acute cellular rejection (ACR), while RV free wall strain $<17 \%$ is related to a significant degree of ACR. ${ }^{7}$ The utility of myocardial deformation using strain measurements of both $\mathrm{LV}$ and $\mathrm{RV}$ in the detection of ACR has been investigated, with promising results. Acute cellular rejection is associated with a significant reduction in peak systolic longitudinal and radial strain. ${ }^{8}$

Apart from RVEF, a quantitative approach to assess $\mathrm{RV}$ systolic function is the measurement of the TAPSE. It is reproducible and proved to be a strong predictor of prognosis in heart failure. Tissue Doppler imaging allows quantitative assessment of RV systolic and diastolic function. Peak systolic velocity $<11.5 \mathrm{~cm} / \mathrm{s}$ identifies the presence of RV dysfunction with a sensitivity and specificity of 90 and $85 \%$ respectively. ${ }^{9}$ Other measures of RV systolic function is IVA (normal $=1.4 \mathrm{~m} / \mathrm{s}^{2}$ ) and diastolic function are tricuspid early (E) to late (A) filling velocity ratio $(\mathrm{E} / \mathrm{A}=1.5)$, isovolumic relaxation time (IVRT $<60 \mathrm{~ms}$ ), and rapid myocardial filling velocity $\left(\mathrm{e}^{\prime}=15.6 \mathrm{~cm} / \mathrm{s}\right){ }^{8,10}$ Some degree of RV dysfunction with reduced tricuspid systolic velocity $(5.8 \pm 1.4 \mathrm{~cm} / \mathrm{s})$ has been shown to not normalize even 5 years after transplantation. ${ }^{11}$ The TDI-derived velocities of the medial and lateral mitral annuli and the tricuspid annulus exhibited a gradual increment during the follow-up up to 4 weeks after transplant. Tissue Doppler imaging should be conducted two to three times each year to survey graft impairment because only in heart transplant patients with graft failure, there is a higher rate of reduction in velocities noticed at early follow-up. ${ }^{10}$ In our case, there was a gradual improvement in the RV systolic function as assessed by TAPSE and TDI-derived peaks' velocity during our 1-month follow-up period.

The assessment of valvular competence reveals TR $>$ mild grade in $38.5 \%$ of the patients on the first posttransplant day and $6.2 \%$ at 5 months' follow-up. ${ }^{10}$ Tricuspid regurgitation is the most common valvular abnormality after orthotropic heart transplant. Its reported overall incidence varies between 19 and $84 \%$. The TR occurring in the early perioperative period is, for the most part, being functional. The regurgitant jet is typically central and caused by geometric distortion of the $\mathrm{AV}$ annular ring and dilation, as well as malcoaptation of the valve leaflets. ${ }^{10}$

Mainstays in the treatment of right ventricular dysfunction are optimizing right ventricular preload, increasing contractility, lowering right ventricular afterload, improving coronary perfusion, and failing these therapeutic interventions mechanical circulatory support. Judicious use of volume therapy is mandatory to avoid volume overload in the postoperative setting. Therapy, in general, is aimed at reducing RV afterload while maintaining an adequate but not excessive RV preload ( $\mathrm{CVP}<15 \mathrm{~mm} \mathrm{Hg}$ ). Positive inotropic therapy 
is indicated to treat hypotension along with right ventricular dysfunction. Dobutamine may be a choice in the presence of a low cardiac index, but preserved systemic pressures and epinephrine in cases of low cardiac output syndrome and systemic hypotension. Low-dose vasopressin $(0.03-0.1 \mathrm{U} / \mathrm{min})$ can be added to $\alpha$-agonist for vasodilatory shock. A useful adjunct to catecholamine therapy is phosphodiesterase-III inhibitors, milrinone in the absence of arterial hypotension. Most importantly, PASP and RV afterload have to be lowered in compromising right ventricular function. Inhaled NO in therapeutic doses selectively dilates the pulmonary vasculature without inducing systemic hypotension. To prevent a rebound phenomenon, inhaled NO therapy has to be slowly weaned. Inhibition of posphodiesterase 5 by sildenafil selectively induces pulmonary vasodilatation without deleterious effects on the systemic circulation. It can also be employed to facilitate weaning heart transplant recipients of inhaled $\mathrm{NO}$, catecholamines, and mechanical ventilator support., 8,12

Impaired right ventricular function of the transplanted heart could be due to various factors but can be managed successfully in the postoperative period. Furthermore, serial strain measurements of RV have good correlation with other measures of RV function and can be used to monitor RV function after heart transplantation.

\section{REFERENCES}

1. Hosenpud JD, Bennett LE, Keck BM, Boucek MM, Novick RJ. The Registry of the International Society for Heart and Lung Transplantation: Seventeenth official report - 2000. J Heart Lung Transplant 2000 Oct;19(10):909-931.

2. Young JB, Leon CA, Short HD, Noon GP, Lawrence EC, Whisennand HH, Pratt CM, Goodman DA, Weilbaecher D, Quinones MA, et al. Evolution of hemodynamics after orthotopic heart and heart-lung transplantation: early restrictive patterns persisting in occult fashion. J Heart Transplant 1987 Jan-Feb;6(1):34-43.
3. Bhatia SJS, Birschenbaum JM, Shemin RJ, Cohn LH, Collins JJ, Di Sesa VJ, Young PJ, Mudge GH Jr, Sutton MG. Time course of resolution of pulmonary hypertension and right ventricular remodeling after orthotopic heart transplantation. Circulation 1987 Oct;76(4):819-826.

4. Hosenpud JD, Norman DJ, Cobanoglu MA, Floten HS, Conner RM, Starr A. Serial echocardiographic findings early after heart transplantation: evidence for reversible right ventricular dysfunction and myocardial edema. J Heart Transplant 1987 Nov-Dec;6(6):343-347.

5. Van Trigt P, Bittner HB, Kendall SW, Milano CA. Mechanisms of transplant right ventricular dysfunction. Ann Surg 1995 Jun;221(6):666-676.

6. Iyer A, Kumarasinghe G, Hicks M, Watson A, Gao L, Doyle A, Keogh A, Kotlyar E, Hayward C, Dhital K, et al. Primary graft failure after heart transplantation. J Transplant 2011;2011:Article ID 175768, 9 pages.

7. Mingo-Santos S, Moñivas-Palomero V, Garcia-Lunar I, Mitroi CD, Goirigolzarri-Artaza J, Rivero B, Oteo JF, Castedo E, González-Mirelis J, Cavero MA, et al. Usefulness of twodimensional (2D) strain parameters to diagnose acute rejection after heart transplantation. J Am Soc Echocardiogr 2015 Oct;28(10):1149-1156.

8. Costanzo MR, Dipchand A, Starling R, Anderson A, Chan M, Desai S, FedsonS, Fisher P, Gonzales-StawinskiG, Martinelli L, et al. The International Society of Heart and Lung Transplantation Guidelines for the care of heart transplant recipients. J Heart Lung Transplant 2010 Aug;29(8):915-926.

9. Bleeker GB, Steendijk P, Holman ER, Yu C-M, Breithardt OA, Kaandorp TAM, Schalij MJ, van der Wall EE, Nihoyannopoulos P, Bax JJ. Assessing right ventricular function: the role of echocardiography and complementary technologies. Heart 2006 Apr;92 (Suppl 1):i19-i26.

10. Behzadnia N, Kashani BS, Mirhosseini M, Moradi A, Radmand G, Ahmadi ZH. Echocardiographic evaluation of orthotopic heart transplantation: single-center experience. Arch Cardiovasc Imaging 2014 Nov;2(4):e24391.

11. FyfeDA,MahleWT,KanterKR, WuG, VincentRN, KetchumDL. Reduction of tricuspid annular doppler tissue velocities in pediatric heart transplant patients. J Heart Lung Transplant 2003 May;22(5):553-559.

12. Wagner F. Monitoring and management of right ventricular function following cardiac transplantation. Appl Cardiopulmonary Pathophysiol 2011;15:220-229. 